\title{
Blockchain Applications and Use Cases in Health Information Technology
}

David Randall*, Pradeep Goel and Ramzi Abujamra

American Research and Policy Institute, Washington, DC, USA

\begin{abstract}
Blockchain technology and the associated crypto currencies have the ability to transform industries including healthcare. We suggest the decentralized and programmable nature of blockchain applications can be used to change health information technology to gain greater efficiency in public and private health care systems. Current public health information technology systems such as eligibility, enrollment and electronic health records have documented issues with interoperability and are slow to adapt to changing program and technology demands. We suggest that blockchain can potentially solve these issues. We argue that a public program such as the U.S. Medicaid program with $\$ 553$ Billion in total program costs and over $\$ 25$ Billion spent on health information technology and administration last fiscal year could benefit from the use of blockchain based distributed ledger and smart contracts. We finally argue that a decentralized benefits administration system can provide greater efficiency to enrollment, eligibility, claims payment and adjudication processes thus driving efficiency and reducing systemic fraud.
\end{abstract}

Keywords: Blockchain; U.S. medicaid; U.S. medicare; Cost containment; U.S. health care reform

\section{Introduction}

Blockchain technology applications have the potential to transform our current use of health information technology and the associated hardware and software infrastructure. The underlying technology and associated crypto currency with its decentralized architecture suggests a range of applications that can, we argue, bring cost savings and efficiencies versus traditional legacy systems currently in use in not only the public healthcare space but also in associated private market participants. We suggest that there are numerous applications that can be implemented in public programs in the U.S. health care system using blockchain technology.

The fundamental promise of the blockchain is the underlying Information Technology (IT) architecture and its 'unbreakable' chain of data entries that allow for secure and open transactions. The decentralized and distributed database of a blockchain that contains data allows for an auditable and distributed ledger that allows all to see every transaction. The open source attributes of the blockchain make the technology a natural fit for the requirements associated with the complexities of transaction laden systems associated with health information technology in the public and private sector.

We suggest that there are specific applications in public programs that include both the U.S. Medicaid and Medicare systems that could benefit from the deployment of blockchain applications by replacing expensive hardware and software IT systems with a blockchain infrastructure. The Medicaid program spent $\$ 553$ Billion in Federal Fiscal Year 2016 with that amount predicted to grow at $5-8 \%$ or more per year [1]. Administrative costs of the Medicaid program are estimated at 5-6\% of total spend or nearly $\$ 30$ Billion, which includes costs associated with health information technology deployment and maintenance [2]. In addition, we argue that the potential cost savings would be significant versus current costs and ongoing system maintenance of existing legacy IT systems. Blockchain is relatively easier to program and to implement system wide changes in comparison to changes made to legacy IT systems.

The advantages of blockchain are obvious, but with any new technology there are questions about efficacy and efficiency. In this paper we attempt to answer questions about the technology, issues of interoperability and specific applications related to the health care space and associated costs. We also address some of the privacy and data security concerns associated with health care that are inherent in any health IT system. Finally, we also examine the policy implications of deploying blockchain technology and suggest further areas of research.

\section{Technology Description and Application}

The advent of blockchain technology can trace its beginnings to creation of Bitcoin as a digital crypto currency [3]. While the rise of digital only currencies in recent years has captured the attention of the financial industry, the public, and regulators it remains largely unknown to the general public. The technology that helped to launch Bitcoin or 'blockchain' is of particular interest and potential use to a wide range of industries, including healthcare because of the open source and decentralized nature of the technology.

Blockchain has potential to improve healthcare in a number of innovative ways. Some of those examples include a Master Patient Identifier (MPI), autonomous automatic adjudication and interoperability [4,5]. MPI's offer a single person identifier that can follow the patient in various situations, enabling a more seamless and scalable health delivery across the continuum of care providers (as well as possibly beyond healthcare where data would also of relevance) [6].

Autonomous automatic adjudication would simplify and lead to significant efficiencies in how claims or other healthcare transactions are processed between parties. Essentially, blockchain could lead to elimination of the third party thus creating a more efficient process. The process would use smart contracts across parties that would enable automatic adjudication of claims. Enhancing claim adjudication in health care has the benefit of reduction in the occurrence of claim fraud that is currently prevalent [5]. All players in healthcare, from providers to payers, would benefit from this reduction in fraud.

*Corresponding author: David Randall, Executive Director and Resident Scholar American Research and Policy Institute, Suite 200, 1250 Connecticut Ave, NW Washington, DC, USA, Tel: (202) 558-6364; E-mail: info@arapi.org

Received July 13, 2017; Accepted July 17, 2017; Published July 20, 2017

Citation: Randall D, Goel P, Abujamra R (2017) Blockchain Applications and Use Cases in Health Information Technology. J Health Med Informat 8: 276. doi: 10.4172/2157-7420.1000276

Copyright: $\odot 2017$ Randall D, et al. This is an open-access article distributed under the terms of the Creative Commons Attribution License, which permits unrestricted use, distribution, and reproduction in any medium, provided the original author and source are credited. 
Fraud detection and mitigation is increasingly becoming a policy issue for state Medicaid programs. Conservative estimates from the U.S. Government Accountability Office (GAO) estimates that fraud in the Medicaid program is over $\$ 14$ Billion per year or $4 \%$ of program spend and expected to grow as program spending increases [7]. Other research finds that Medicaid fraud could be as high as $15-22 \%$ of total spend as a result of over billing for services $[8,9]$. These findings suggest that the deployment of a blockchain application that utilizes a smart contract and verifiable ledger of all service and payment activities could reduce fraud and overpayment that is prevalent in the U.S. Medicaid system.

Blockchain could also improve interoperability across systems and organizations. This is crucial for progress in our current health ecosystem which consists of a plethora of disparate IT legacy systems that have been amassed over the years and that do not communicate well with each other. Blockchain would provide the ability to replace these disparate systems with a single system that offers interoperability. With the use of smart contracts and fully auditable history, Blockchain would enable peer-to-peer interoperability among participants within transactions. [16] In addition to offering interoperability, blockchain transactions would also have the advantage of being cryptographically and irrevocable thus ensuring privacy across parties. The patient would be able to designate by whom the data can be accessed (and at what level of access) by the use of keys that users would have access to (either private or public) [5].

The benefit of blockchain in healthcare would take place across the entire supply chain spectrum. Contractual agreements between payer and patient or provider and patient (or between provider and payer) would be implemented with the use of smart contracts within the blockchain [10]. Smart contracts lead to efficiencies as they enable a reduced number of intermediaries that exist today which lead to more streamlined transactions [11]. These transactions would enable a more holistic view of the patient's record for all parties involved and lead to an increase in transparency. Beyond contractual transactions, clinical transactions based on electronic health records would also occur on the blockchain. This would enable clinicians to have access to different components of the patient's data throughout the patient's lifetime with an increase in transparency (this access would be controlled so that providers have access to data only on a need-to-go basis) $[12,15]$. Improvements in the supply chain from blockchain would be extended to drug companies and manufacturers, as well as improvement to pharma clinical trials and longitudinal health research for the patient $[5,16]$.

The federal government can also benefit from the improvement in blockchain with more streamlined transactions in the supply chain. For instance, one of the issues facing Medicaid recipients is high churn due to changing economic qualifications. Blockchain can be helpful to maintain a recipient's identity as they pivot between different governments systems in a more seamless way [17]. This would be especially valuable if account based plans from the private sector are implemented as an option for Medicaid recipients (with hope of reduction in costs and better outcomes) [18]. Other government programs would similarly benefit from a more streamlined delivery of care across all players within the supply chain, including with private prescription plans [10].

Overall, blockchain applications have been surging in a number of different industries. According to Deloitte, $35 \%$ of health and life sciences companies surveyed plan to use blockchain, and $28 \%$ of respondents plan to invest $\$ 5$ million or more (10\% plan $\$ 10$ million or more) [19]. In finance, it is expected that savings from reduced intermediaries (and slow payment networks) has potential savings of $\$ 15-20$ billion by year $2022[10,12]$. The industries implementing blockchain are numerous and are led by real estate, supply chain and others. Within healthcare, a number of companies have begun implementations of blockchain in various areas of applications-including Gem (in collaboration with Philips Healthcare Blockchain Lab), PokitDok, Healthcoin, Hashed Health and many others [4]. The Hyper ledger Healthcare Working Group is a consortium that provides an open source collaboration of member companies in healthcare with the goal to speed the development of commercial adoption of blockchain $[16,20]$.

The applications of blockchain technology are numerous as well the potential to transform legacy health information technology. We focus our discussion on public health information technology because of not only its impact on the U.S. healthcare system but also the complexities associated with integration and deployment that we believe deployment of blockchain technology can have not only ease of development and maintenance but also cost savings versus current IT infrastructure.

\section{Records Management}

The promise of universal provider adoption of electronic records systems has been a 'holy grail' for the health information technology market. Recent industry and government reports suggest that adoption of electronic health record standards outlined by the 2009 law that has funded much of the industry growth is lagging behind with a good deal of variation in provider adoption rates. These facts take on added significant as the Centers for Medicare and Medicaid Services (CMS) begin to tie future funding to provider adoption and 'meaningful use' attestation by providers. Given these coming milestones and varying degrees of implementation success, serious questions remain about the future of wide-spread adoption of electronic health records systems and how the U.S. healthcare system will look in the coming decade [21]

The U.S. Recovery Act of 2009 provided an enormous financial boost toward adoption of electronic health records with over $\$ 34$ Billion appropriated to fund provider information technology hardware and software solutions [22]. As implementation has progressed, there are numerous milestones established by both the authorizing statute, but also under CMS guidelines and regulations. The so called 'meaningful use' guidelines are set in series of stages, with Stage 1 designed to facilitate data capture and sharing, which ended in 2012. Stage 2 of the program intended to advance clinical processes for all eligible healthcare professionals and hospitals and to report to CMS by the end of 2014 . The final Stage 3 of the program was slated to be fully implemented by 2016 with goal of improved clinical outcomes as result of Electronic Health Records (EHR) use by providers, but has been delayed [23]. Each stage of the implementation process has had varying degrees of success with both hospitals and provider professionals as evidenced by both published research and various industry reports. The variation in adoption rates of EHR standards raises the greatest levels of concern among health IT professionals, policy makers and academics about the prospects for wide adoption of uniform electronic standards.

Adoption rates and the progress toward Stage 3 EHR standards continue to be of concern to not only providers, but policymakers as well. Recent reports suggest that while EHR use has grown from $20 \%$ of providers using the technology in 2002 to over $60 \%$ today, much of the increased use has been uneven across professional providers and hospitals. Rural hospitals have generally lagged their peers and older physicians (greater than age 55) have lower adoption rates [24]. Given these lower than expected adoption rates, many health IT firms have struggled to achieve CMS guidelines with their clients as many small 
providers struggle to implement Stage 2 standards, which leaves serious questions about how the majority of professional providers can achieve Stage 3 attestation and meaningful use standards [21].

Federal government agencies are not immune to the challenges of implementing EHR standards. Both the Veteran's Administration and the Department of defence have had numerous implementation issues in their attempt to use EHR technology and to increase the interoperability of their respective systems [25]. Government records management in general and EHR specifically suggest that the deployment of a blockchain solution could potentially alleviate the issues discussed. Most notably the issues surrounding interoperability and secure data access can be achieved through APIs in a blockchain environment.

\section{Medicaid Management Information Systems}

The Affordable Care Act authorized the US Department of Health and Human Services (HHS) and the Centers for Medicare and Medicaid Services (CMS) to create health information technology systems to assist in the implementation of the programs created under the law. Central to the architecture of the ACA is the coordination of diverse set of disparate programs (including Medicaid and various social programs) and accompanying legacy technology systems that are needed to verify eligibility, enrolment and ongoing programmatic support. Inherent in the implementation of these ACA programs are the varied nature of state policy choices and associated complexity [26].

In anticipation of the passage of the Affordable Care Act, CMS developed and codified a policy and financing structure designed to provide states with tools needed to achieve the immediate and substantial investment in information technology systems. In order to achieve this goal, federal funding is provided through a variety of venues to help states improve their eligibility and enrolment systems.

Central to the implementation process is the creation of a Medicaid Information Technology Architecture (MITA). MITA is a national framework to support improved systems development and health care management for the Medicaid enterprise. MITA has a number of goals, including development of seamless and integrated systems that communicate effectively through interoperability and common standards. The MITA standards are a critical standard for states in linking the complex systems associated with Medicaid Management Information Systems or MMIS [27].

The MMIS is an integrated group of procedures and computer processing operations (subsystems) developed at the general design level to meet principal objectives. For Title XIX purposes, "systems mechanization" and "mechanized claims processing and information retrieval systems" is identified in section 1903(a)(3) of the Act and defined in regulation at 42 CFR 433.111. The objectives of this system and its enhancements include the Title XIX program control and administrative costs; service to recipients, providers and inquiries; operations of claims control and computer capabilities; and management reporting for planning and control. States may receive 90\% Federal Financial Participation (FFP) for design, development, or installation, and $75 \%$ FFP for operation of state mechanized claims processing and information retrieval systems approved by the secretary [27].

The MMIS program spent $\$ 3.7$ Billion in 2015 and total administration and other technology spend on eligibility systems, electronic health records and technology associated with administration was over \$25 Billion in 2015 [13]. Often, there are integration and interoperability issues that cause significant cost overruns and additional maintenance costs to both the state and federal government. The promise of blockchain can potentially solve these issues at a reduced cost due to relative ease of deployment versus traditional hardware and software infrastructure. We argue that the MMIS program is a candidate for the deployment of blockchain to only reduce costs but gain additional efficiency to deal with the inevitable changes in the program and technology.

\section{Benefits Administration}

Blockchain technology has the ability to simplify and reduce the cost of benefits administration. As benefits design, enrolment, beneficiary engagement and provider payment systems have evolved overtime there are the inevitable and predictable issues associated with the myriad of health information technology systems designed to work together. Interoperability has become a key concern and challenge in the development and deployment of the health IT infrastructure. We suggest that the use of blockchain application can assist in dealing with the challenges and allow for potentially infinite modularity and allow multiple systems to work in greater efficiency. Benefits administration involves the use of disparate systems that are designed to gather and process data from numerous sources. This fact is especially true in public health care systems, including Medicaid and Medicare as well as Health Insurance Exchanges administered by the states and the U.S. federal government. Integration complexity is a hallmark of these systems as previously discussed.

The challenges associated with blockchain deployment with any complex health care system not only include interoperability, but also issues associated with data access and privacy [14]. Data privacy and the ability to access sensitive patient specific information is one of the key challenges in the design of a healthcare blockchain application. Numerous researchers and software engineers suggest that addressing authentication of users can be achieved through design of systems that utilize a Proxy pattern to facilitate the transfer of data [15].

The promise of using blockchain applications are readily apparent when considering how the deployment of the technology can be flexible and address key issues of interoperability and data privacy. There are numerous processes and systems that potentially meet the criteria and challenges with the new technology; this includes the use of benefit incentive systems such as wellness programs, enrolment and eligibility systems and the previously discussed records management systems surrounding EHR deployment.

Current benefits administration systems suffer from several key limitations that we argue are a direct result of the centralized nature of health information technology today. We also suggest that existing large scale health IT infrastructure are inflexible and costly to maintain and update to deal with programmatic technology changes. As an example, we argue that current eligibility and enrolment systems have rigid logic rules that often cannot deal with the inevitable issue with beneficiaries and their needs. This fact is largely a result of centralized networks that are often inflexible.

The centralized benefit administration is synonymous with data silos that are designed to keep people out and data secure, which are admirable attributes in today's cyber security environment but also prevent personalization of benefits and reduce timely coordination among various stakeholders. We suggest that the documented issues of duplication, fraud, and overutilization of service are traceable to the need for data security and data availability. The centralized nature of current systems lead to a limited and narrowly defined views of what 
data needs to be shared by whom to whom and who owns the data. We argue that the data belongs to the individual, not the system nor the corporate entities that build the system. We believe that a blockchain application environment utilizing smart contracts can remove many of the barriers and limitations inherent with centralized technology systems.

The potential impact of decentralized benefit and care management system could have numerous benefits to the health care system. We suggest that the impacts would include instant eligibility and recognition of the benefits through the use of a smart contract. Additionally, a blockchain application could provide an unbreakable chain that would allow for individualized care through smart care contracts that do not break administrative or clinical systems. We believe that the convergence of clinical and administrative systems that can be individualized through a blockchain smart contract would reduce the duplication inherent in centralized data silo systems.

The deployment of all the needed applications in a blockchain environment would naturally require the use of portals that would allow each of the needed systems to communicate. As discussed in addressing the use case for records management, interoperability becomes a prime challenge. The development of a so-called 'smart benefit' design system we suggest can be achieved through blockchain applications that work together through the use of smart contract design similar to the Ethereum model with modifications that allows for the inclusion of HIPAA compliant privacy protocols that providers can modify data of patients via specific permissions.

The potential for the use of an integrated approach and associated challenges are issues that be solved through further software development and the use of a blockchain application protocol that allows for scalability, interoperability and unique patient and provider access. The ability for the applications to be easily modified we suggest will lead to significant cost reductions in not only IT system integration but also ongoing maintenance and modifications that offer the ability of blockchain to be far more flexible than current health information technology systems.

\section{Policy Implications and Conclusions}

We argue that blockchain technology has the ability to address the documented shortcomings of public and even private legacy health information technology systems. In each of the above addressable programs and markets core issues of interoperability, data access and privacy and the ability to adapt to changing programs and technology are potentially solved through the use of Blockchain applications. We suggest that deployment of blockchain applications be incremental in nature as with any emerging technology.

As discussed, federal and state agencies are relatively slow to adopt and adapt large scale information technology changes. This is evidenced by the both the EHR rollout by the Veteran's Administration as well the processes that CMS utilizes for MMIS, which are understandable and somewhat rationale by policymakers given the scale and scope of populations being served. We argue as with Defense Advanced Research Projects Agency (DARPA) government examine selective system implementation of blockchain that can achieve elusive program goals of interoperability, flexibility and needed privacy considerations through experimental programs that focus on research that can quantify functionality of the technology and cost savings associated with deployment and ongoing maintenance costs.
We suggest that further research should focus on costs associated with deployment, ongoing maintenance of systems and the ability of systems integrators to adapt to changing programmatic mandates as well as technology innovation. Additionally, we suggest that use of multiple types of applications across varying program functions would be useful to examine as we have described in this paper. As with any new technology, there will be undoubtedly be issues in deployment and implementation but we believe that given the adaptive nature of the blockchain and the ability to make changes in applications that efficiency and efficacy will demonstrate the possibilities we discuss.

\section{References}

1. http://www.kff.org/medicaid

2. http://www.kff.org/report-section/medicaid-financing-how-does-it-work-andwhat-are-the-implications-issue-brief/\#endnote_link_152625-6

3. http://www.bitcoin.org/bitcoin.pdf

4. https://www.linkedin.com/pulse/how-blockchain-can-solve-real-problemshealthcare-tamara-stclaire

5. https://www.forbes.com/sites/reenitadas/2017/05/08/does-blockchain-have-aplace-in-healthcare/\#6afb55a71c31

6. https://www.healthit.gov/sites/default/files/11-74-ablockchainforhealthcare.pdf

7. http://www.gao.gov/assets/670/668233.pdf

8. Parente ST, Oberlin S, Tomai L, Randall D (2016) The Potential Savings of Using Predictive Analytics to Staunch Medicaid Fraud. J Healt Med Econom 2:2.

9. http://ehrintelligence.com/2012/04/25/ehr-adoption-depends-on-age-grouppractice-size-and-setting-and-specialty/

10. https://www.linkedin.com/pulse/blockchain-transformational-technologyhealth-care-bruce-broussard?trk=vsrp_people_res_infl_post_title

11. https://www.healthit.gov/sites/default/files/8-31-blockchain-ibm_ideation challenge_aug8.pdf

12. http://www.coindesk.com/santander-blockchain-tech-can-save-banks-20billion-a-year/

13. MACPAC (2016) MACStats: Medicaid and CHIP Data Book pp: 78-81.

14. http://www.healthcareitnews.com/news/blockchain-faces-tough-roadblockshealthcare

15. Zhang P, White J, Schmidt DC, Lenz G (2017) Applying Software Patterns to Address Interoperability in Blockchain-based Healthcare Apps 24: 22-25.

16. http://www.healthcareitnews.com/node/53648

17. https://www.healthit.gov/sites/default/files/14-38-blockchain_medicaid_ solution.8.8.15.pdf

18. Randall D, Parente ST, Abujamra R (2015) Medicaid Expansion and the use of Account-based Health Plans. Health Care Curr Rev 3:134.

19. http://www.healthcareitnews.com/news/how-does-blockchain-actually-workhealthcare

20. http://www.hyperledger.org/

21. Ramlet M, Randall D, Parente ST (2013) Insurer Payment Lags to Physician Practices: An Opportunity to Finance EMR Adoption. J Healt Med Informat 4: 136.

22. http://www.gpo.gov/fdsys/pkg/PLAW-111publ5/html/PLAW-111publ5.htm

23. http://healthit.gov/providers-professionals/meaningful-use-definition-objectives

24. Decker S, Jamoon E, Sisk J (2012) Physicians in Nonprimary Care and Small Practices and Those Age 55 and Older Lag in Adopting Electronic Health Record Systems. Health Affairs 31: 1108-1114.

25. http://www.healthcareitnews.com/news/congress-demands-va-dodinteroperability-progress

26. Randall, D, Parente ST (2012) US Medicaid Managed Care Markets: Explaining State Policy Choice Variation. Insurance Markets and Companies: Analyses and Actuarial Computations 3: 35-49.

27. https://www.medicaid.gov/medicaid-chip-program-information/by-topics/dataand-systems/mmis.html 